schon fünf Tage später folgte C. seiner Gattin im Tode. Seine Grabstätte hatte er längst im Chorherrenstift Hof erworben. Die Regierung ehrte sein Andenken durch das Geschenk eines «hundertlötigen Silbergeschirrs» an seine Hinterbliebenen. A. von HaLler hat ihn als den großen CySAT gefeiert.

\title{
Thomas Harriot (1560-1621) fumeur de pipe, victime du cancer?
}

Par JuraJ Körbler, Zagreb

Déjà en 1739 Johann Jacob Holland supposait dans sa dissertation Dissertatio inaugur. med. chir. sistens Carcinoma labii inferioris absque sectione persanatum, qu'il y a une relation causale entre l'usage de fumer la pipe et l'apparition du cancer de la lèvre inférieure. En 1795 Samuel Thomas Soemmering (1755-1830) précise que ce n'est pas la fumé qui provoque la cancerisation mais les sucs qui se forment dans la pipe. R. Melzer ${ }^{1}$ croit avoir observé, qu'une telle cancerisation n'arrive jamais dans l'Orient ou on fume le tabac cru sans aucune préparation préalable. Une observation qui est d'ailleurs tout autre que juste. Il est intéressant de noter à ce sujet, que la première observation d'un cancer de la lèvre inférieure comme suite de l'usage de fumer la pipe est bien plus ancienne. Elle date de la même époque comme l'usage même de fumer la pipe en Europe, cependant la relation existante entre la pipe et le cancer n'était pas encore reconnue. Il s'agit du cancer de la lèvre inférieure dont était atteint Thomas HARriot, savant naturaliste anglais. BERnARD JAFFe donnait récemment un éloge de HARriot dans son livre sur les savants americains: Men of science in America ${ }^{2}$.

L'intérêt des Anglais pour les pays d'outre-mer apparaissait relativement tard. Les Espagnols possédaient déjà à cette époque des empires immenses sur le nouveau continent. Après Humphry Gilbert, qui périt en mer sans avoir réussi de fonder une colonie en Amérique du Nord, son demi-frère Sir Walter Raleigh obtenait le 25 mars 1584 de la reine Elisabeth un patent et une licence pour la colonisation dans le Nouveau Monde. C'est pourquoi Raleigh est consideré comme un des "founders of America".

Raleigh organisa une expédition sous le commandement de Amados et BARLow pour explorer les régions entre Florida et New Foundland. Avec des rapports enthousiastes sur les richesses de ces pays, cette expédition à son retour en Europe amenait deux Indiens, Manteo et Wanchese, pour s'en servir des interprètes dans les expéditions qui devront suivre. Pour mieux explorer tous les richesses de

1 Jenaische Annalen 2, 480 (1850).

${ }^{2}$ New York (1944). 
ces terres vierges auxquelles Raleigh donna le nom Virgina en honneur de sa reine et sa bienfaitrice, RALEIGH cherchait l'appui des savants. Dans ce but son choix tomba sur un jeune naturaliste Thomas Harriot, né en 1560 à Oxford HaRriot entrait à l'âge de 25 ans dans le service de Raleigh, après avoir été gradué à l'Université de Oxford à l'âge de vingt ans.

Le 9 avril 1585 sept vaisseaux démarraient de Plymouth vers le Nouveau Monde. C'était Sir Richard Grenville qui commandait la flotte en qualité d'amiral au nom de Raleigh. Thomas Harriot se trouvait parmi les 107 personnes de l'équipage. La flottille suivait la route de CoLomb et accostait après un voyage de 75 jours à l'île de San Domingo. D'ici on se dirigait vers l'île Roanoke et sur cette île l'équipage érigait une fortresse. Avec l'aide des Indiens Manteo et WaNGHESE, qui accompagnaient l'expédition pour servir des interprètes, HaRriot commançait ses explorations. La paix ne durait pas longtemps. Un chef indien, nommé Pemisapan, soulevait les tribus Indiens des environs contre les nouveau venus et les hostilités commençaient.

Bientôt la faim se faisait sentir dans la colonie comme suite des hostilités. RALPH LANe, qui administrait la colonie après le départ de la flotille, se vut obligé d'envoyer des vedettes au fourage. Des autres vedettes observaient la mer à la recherche d'un vaisseau qui pourrait par un hasard heureux passer près de l'île. Quelques jours après que le chef indien fut tué dans un combat, accostait à l'île Sir Francis Drake qui était en croisière dans ces régions. Les colonistes épuisés par la lutte et par la faim décidaient de retourner en Europe. Le 19 juin 1586 cette «première colonie» fut abandonné et DrAKE amenait les colonistes en Angleterre.

Deux semaines après que la colonie fut abandonné revenait Sir Richard GreNVILLE à l'île pour y déposer des nouveaux colonistes avec des grandes provisions. Ces colonistes périrent tous et tous les détails sur leur sort restent inconnus. Parmi ces colonistes se trouvait Virginia Dare, premier enfant des parents anglais né dans le Nouveau Monde.

Après son retour en Europe Harriot avait décrit ses explorations dans un livre intitulé: $A$ brief and true report of the New Found Land of Virginia. Il avait apporté en Angleterre le maïs (Zea mays), appelé par les indigèns pagatur et déjà bien connu aux Espagnols qui l'avaient trouvé en Pérou. Mais plus que le maĩs attirait l'attention une autre plante apporté par HARRIoT, qui fut nommé par les indigènes uppowoc. Cette plante, le tabac, était, elle aussi, déjà connue aux Espagnols qui la utilisaient seulement pour embellir leurs jardins. Harriot a décrit que les indigènes séchent les feuilles de cette plante et ensuite les pulvérisent. La fumé, qui se développe quand ces feuilles pulvérisées sont allumées, inhalent les indigèns par les pipes d'argile. La fumé pénétre d'après l'opinion de Harriot dans leurs estomacs et leurs têtes d'ou elle purge la chaleur superflue et autres humeurs visqueuses et nocives. 
De plus Harriot attribuait au tabac des qualités salutaires: il ouvre les pores et les passages dans le corps humain. Non seulement qu'il préserve la santé mais il possède même le pouvoir de guérir beaucoup des maladies autrement incurables. Harriot lui même, après avoir appris des indigènes de l'île de Roanoke de fumer la pipe, est devenu un fumeur passioné. De même Raleigh s'enthousiasmait pour le tabac et introduisait à la cour du Saint James la méthode indienne de fumer la pipe d'argile. On sait que déjà Cоцомв avait observé les Indiens qui inhalaient la fumé des feuilles pliés et allumées à une extremité. A la cour de France on fumait déjà en 1560 où le tabac fut apporté de Portugal par l'ambassadeur Français Jean Nicot.

Harriot ne trouvait plus d'occasion de rentrer dans le Nouveau Monde puisque, peu de temps après son retour en Angleterre, RALEIGH avait vendu sa licence à une compagnie commercielle. HARriot restait en Angleterre pour y poursuivre ses recherches scientifiques, sa pipe toujours allumée. En 1607 il observait le comet connu plus tard sous le nom de Halley. Il construisait un télescope, correspondait avec Kepler et Galilei, s'occupait des mathématiques, c'est lui qui était le premier à indiquer la multiplication par un point.

HARRiot restait fidèle à son protecteur RALEIGH même quand celui-ci fut emprisonné avec son ami Earl of Northumberland, accusé par le roi JAMES ${ }^{\text {er }}$ de haute trahison. Dans la geôle ils s'occupaient tous les trois des expérience chimiques. Même dans sa dernière nuit avant de monter sur l'échaffaud en 1618, RALEIGH dictait à son ami HARRIOT la «Note of Remembrence» pour son oraison sur l'échaffaud.

Le 2 juillet 1621 est mort HARriot. Il fut enterré à Londres à la cimetière de Saint Christopher, là où se trouvent aujourd'hui les parcs de la Banque d'Angleterre. Il est mort d'un cancer de la lèvre inférieure! Ces contemporaines attribuaient l'apparition du cancer à son usage de tenir entre les dents ses instruments mathématiques construits en bronze. Personne n'accusait son habitude de fumer la pipe et pourtant d'après l'opinion de nos jours il faut plutôt considerer la maladie de Harriot comme un cancer de fumeurs de pipe. Probablement le premier qui fut observé en Europe, mais pas reconnu comme tel, était le cas de Thomas Harriot. 